\title{
BMJ Open Implementation and impact of mobile health (mHealth) in the management of diabetes mellitus in Africa: a systematic review protocol
}

\author{
Franklin Okechukwu Dike (D) ,' Jean Claude Mutabazi, ${ }^{2,3}$ \\ Blessing Chinenye Ubani, ${ }^{1,4}$ Ahmed Sherif Isa, ${ }^{5,6}$ Chidiebele Ezeude, ${ }^{7,8}$ \\ Ezekiel Musa, ${ }^{9,10}$ Henry Iheonye, ${ }^{11}$ Isah Idris Ainavi ${ }^{12,13}$
}

To cite: Dike FO, Mutabazi JC, Ubani BC, et al. Implementation and impact of mobile health (mHealth) in the management of diabetes mellitus in Africa: a systematic review protocol. BMJ Open 2021;11:e047556. doi:10.1136/ bmjopen-2020-047556

- Prepublication history for this paper is available online. To view these files, please visit the journal online (http://dx.doi org/10.1136/bmjopen-2020047556).

Received 02 December 2020 Accepted 24 November 2021
Check for updates

(C) Author(s) (or their employer(s)) 2021. Re-use permitted under CC BY-NC. No commercial re-use. See rights and permissions. Published by BMJ.

For numbered affiliations see end of article.

Correspondence to

Dr Franklin Okechukwu Dike; frankincense4m@uuthuyo.net

\section{ABSTRACT}

Introduction The WHO has proposed the concept of mobile health (mHealth) to support healthcare systems delivery worldwide. mHealth basically involves the use of Information and Communication Technology for healthcare provision or delivery services. Africa has seen a remarkable increase in mobile phone availability and usage in the last decade. The incidence and prevalence of diabetes mellitus (DM) in Africa have also been on the increase in the last decade, in sharp contrast to an ailing healthcare system. We aim to review the extent of implementation of mHealth in the management of DM in Africa, and estimate its impact in helping patients achieve desired glycaemic target, sustain control and prevent complications in the past decade.

Methods and analysis Studies assessing the utilisation of mhealth in the management of patients with DM in Africa will be considered based on the PICO method: Population, Intervention, Comparator, and Outcomes. Medline, PubMed, SCOPUS and the Pan African Clinical Trials Registry, among others will be searched. Two authors independent of each other shall screen titles and abstracts retrieved using the search strategy, retrieve the full text articles and assess them for eligibility and extract data. A third reviewing author will be brought in to resolve any disagreement between the two authors by discussion. The 'Cochrane Collaboration Risk of Bias Tool' will be used to assess the quality of included studies. A narrative synthesis of extracted data and, where the characteristics of the eligible studies permit, a meta-analysis (which will be reported according to the Preferred Reporting Items for Systematic Reviews and Meta-Analyses guidelines) will be done.

Ethics and dissemination No ethical approval will be required since only published data will be used. Dissemination of results will be through peer reviewed publication and conference presentation.

PROSPERO registration number CRD42021218674.

\section{INTRODUCTION}

In 2009, the Global Observatory for eHealth (GOe) a subsidiary of the WHO devoted an entire section of its global survey to mobile health (mHealth). ${ }^{1}$ The survey sought
Strengths and limitations of this study

- We intend to detect as many eligible studies as possible using our proposed search strategy to search through the largest online databases and libraries.

- We anticipate a lot of heterogeneity during data extraction and analysis due to the large number of mobile health categories available for implementation.

- Reviewers will not be blinded during the data extraction and quality assessment stages.

to determine the extent of adoption of mHealth interventions/initiatives, the types of initiatives, the status of evaluation and the barriers to implementation across 112 member states. ${ }^{1}$ mHealth was defined by GOe as medical and public health practice supported by mobile devices such as mobile phones, patient monitoring devices, personal digital assistants and other wireless devices. ${ }^{1}$ mHealth basically involves the use of Information and Communication Technology for healthcare provision or delivery services. This concept mainly involves the employment of the basic functionality of mobile phones in the delivery of healthcare services and these functions include voice calls and short messaging services also known as Short Messaging Services (SMS), as well as more complex functionalities and applications such as Global Positioning System (GPS), General Packet Radio Service (GPRS) and also third and fourth generation telecommunications 3G, 4G and Bluetooth technology either as part of the mobile phones or as independent wireless devices. ${ }^{1}$

This survey was a follow-up to an earlier survey in 2005. It showed that mHealth was only just emerging in many member states, through experimentation in their health sectors. Greater strategic implementation was 
observed in high-income countries, with up to $87 \%$ implementation of various mHealth categories, compared with low-income and middle-income countries (LMIC) with about $77 \%{ }^{2}$ Most LMICs could only boast of one mHealth intervention being implemented at the time. ${ }^{1}$ Africa had the lowest number of responders in that survey, and the lowest mHealth usage, with a mobile phone penetration of $47 \% .^{2}$

The past decade has seen a revolutionary increase in the penetration and usage of mobile phones in Africa. A recent country by country survey showed a $91 \%$ penetration in South Africa, $80 \%$ penetration in Nigeria and $75 \%$ penetration in Tanzania which had the lowest. ${ }^{3}$ It also demonstrated an increasing adoption of smartphones in many countries, even though it remains the region with the lowest rate of smartphone ownership and usage globally. ${ }^{34}$

Intriguingly, there has been a phenomenal increase in the prevalence of non-communicable diseases (NCDs) in Africa. ${ }^{5}$ This in addition to the already high prevalence of infectious diseases notably malaria, HIV and tuberculosis results in a double burden of diseases in Africa. This epidemiological transition has witnessed a rising incidence of diabetes mellitus (DM) and hypertension across populations in Africa. ${ }^{5}$ The transition is thought to be a result of urbanisation, an increasing adoption of western diets, sedentary lifestyles (which result in obesity), and advancing age. ${ }^{67} \mathrm{DM}$ remains one of the most expensive chronic diseases in Africa and world-wide. ${ }^{8}$ Prevention of diabetes and improving self-management among people with diabetes are some of the main priorities of diabetes as a public health problem.

mHealth has theoretically been thought to be suitable as a supportive intervention in the management of DM to help patients achieve desired target, sustain control and prevent complications. ${ }^{9}{ }^{10}$ A 2011 meta-analysis of 1657 individuals with type 1 or type 2 DM using SMS messages to send self-monitored blood glucose values and receive self-management information actually showed a $0.5 \%$ reduction in glycated haemoglobin (HbA1c) over a period of 6 months in mHealth intervention groups compared with control groups. ${ }^{10}$ More recently in 2017, another meta-analysis which included 13 studies reported a mean reduction of $0.44 \%$ in $\mathrm{HbA1c}$ in mHealth intervention groups compared with controls. ${ }^{10}$ Furthermore, it reported an increased perception of self-care. ${ }^{10}$ The WHO recommends a HbAlc glycaemic target of less than or equal to $7 \%$ for most patients with type $2 \mathrm{DM}^{11}$

Thus, the burgeoning increase in the usage of mobile phones across Africa is a welcome development in terms of the potential it holds with regards to supporting existing healthcare systems in combating both communicable and NCDs. In the absence of a more recent global survey, it is still pertinent to focus on Africa, and review its adoption and implementation of mHealth strategies to support most of its ailing healthcare delivery systems, and probably identify any impeding factors. Currently, there are no data showing the degree of implementation of mHealth in Africa as recommended by WHO-GOe, especially regarding management of DM. While a systematic review of economic evaluation of mHealth solutions from studies conducted mostly in upper and upper-middle income countries concluded that mHealth is cost effective, ${ }^{12}$ same cannot be said for the African continent due to unavailability of data. Reported harms from mHealth range from loss of privacy, to loss of reputation, poor quality of data, poor lifestyle/clinical decision, inappropriate but reversible clinical action and inappropriate and irreversible clinical actions. ${ }^{13}$ This study aims to review the extent of implementation of mHealth in the management of DM in Africa, and estimate its impact in the past decade. The outcome of this systematic review will provide data that will advise clinicians, diabetes nurse educators and dieticians on the benefits and compelling need for implementation of mHealth. It will also inform some policy framework to embed mHealth in the standard of diabetes care in Africa.

\section{OBJECTIVES}

1. To determine the extent of implementation of mHealth strategies, and the common forms of mHealth used in the management of DM in patients in Africa.

2. To determine the impact/efficacy of mHealth interventions in helping patients achieve treatment goals in diabetes in Africa.

3. To determine the impact of mHealth on early detection, management and outcome of some diabetic complications.

4. To identify any factors impeding the utilisation and implementation of mHealth in Africa in management of patients with DM.

5. To determine the possible harms and cost of implementation of mHealth strategies in Africa.

\section{METHODS}

\section{Eligibility criteria}

1. All published studies deploying one or more mHealth strategies in the management of DM done in the continent between January 2010 and October 2021, including randomised clinical trials and non-randomised trials, quasi-randomised clinical trials and observational studies. Unpublished studies from the Pan African Clinical Trials Registry (PACTR) shall also be included.

2. Diagnosis of DM and treatment targets (fasting plasma glucose and $\mathrm{HbA1c}$ ) are based on the guidelines of the WHO and the International Diabetes Federation (IDF).

3. No language restrictions.

\section{Exclusion criteria}

1. Studies testing acceptability of mHealth among health professionals will be excluded.

2. Duplicate publications. 
Table 1 Search strategy

\section{Implementation and impact of mobile health (mHealth) in the management of diabetes mellitus in Africa}

\begin{tabular}{|c|c|c|c|c|c|c|}
\hline $\begin{array}{l}\text { (implement }{ }^{\star} \text { OR } \\
\text { execute* OR } \\
\text { administrate* OR } \\
\text { organize* OR fulfill* }^{\star} \\
\text { OR perform* OR } \\
\text { utilize* OR manage }^{\star} \\
\text { OR operat* OR act* } \\
\text { OR realiz* OR effect* } \\
\text { OR impact*) }\end{array}$ & AND & $\begin{array}{l}\text { (mHealth* OR } \\
\text { "mobile health*" } \\
\text { OR eHealth* OR } \\
\text { "electronic health*") }\end{array}$ & AND & $\begin{array}{l}\text { (diabetes* } \\
\text { OR diabetes } \\
\text { mellitus, type } \\
\text { 1/OR diabetes } \\
\text { mellitus, type } \\
2 \text { OR diabetes, } \\
\text { gestational/) }\end{array}$ & AND & $\begin{array}{l}\text { Africa/ OR "Africa South of the Sahara"/ OR } \\
\text { "Sub-Saharan Africa"/ OR north Africa/ OR } \\
\text { Africa, Northern/ Egypt or Libya OR Tunisia OR } \\
\text { Algeria OR Morocco OR "Western Sahara" OR } \\
\text { Angola/ OR Benin/ OR Botswana/ OR Burkina } \\
\text { Faso/ OR Burundi/ OR Cameroon/ OR Cape } \\
\text { Verde/ OR Central African Republic/ OR Chad/ } \\
\text { OR Comoros/ OR Congo/ OR Brazzaville/ } \\
\text { OR Cote d'Ivoire/ OR Djibouti/ OR Equatorial } \\
\text { Guinea/ OR Eritrea/ OR Ethiopia/ OR Gabon/ } \\
\text { OR Gambia/ OR Ghana/ OR Guinea/ OR } \\
\text { Bissau/ OR Kenya/ OR Lesotho/ OR Liberia/ } \\
\text { OR Madagascar/ OR Malawi/ OR Mali/ OR } \\
\text { Mauritania/ OR Mauritius/ OR Mozambique/ } \\
\text { OR Namibia/ OR Niger/ OR Nigeria/ OR } \\
\text { Rwanda/ OR Sao Tome e Principe/ OR } \\
\text { Senegal/ OR Seychelles/ OR Sierra Leone/ OR } \\
\text { Somalia/ OR South Africa/ OR South Sudan/ } \\
\text { OR Sudan/ OR Swaziland/ OR Eswatini OR } \\
\text { Tanzania/ OR Togo/ OR Uganda/ OR Western } \\
\text { Sahara/ OR Zaire/ OR Zambia/ OR Zimbabwe/ }\end{array}$ \\
\hline
\end{tabular}

\section{SOURCES OF INFORMATION}

We shall search the following electronic databases for relevant studies implementing mHealth for DM in Africa published between January 2010 and October 2020: Medline database (PubMed), Cochrane Register of Controlled Trials (CENTRAL) and Embase (Scopus).

The PACTR shall also be searched for on-going studies that meet the criteria, as well as recently completed but unpublished studies.

\section{SEARCH STRATEGY}

Medical Subject Headings (MeSH) will be used to search through Medline database for all available studies on the subject (table 1).

Terms describing all the available m-Health categories or relating to them will also be used in the MeSH search, using DM and Africa (regions and countries) as further filters. All available controlled trials using this search will be retrieved.

Similar methods will be used in searching through the CENTRAL, and Embase (Scopus).
Hand searching and snowballing will be used when necessary through all the databases to retrieve all relevant articles.

Data from dissertations that are indexed will be retrieved where possible. The PACTR will also be searched for any ongoing, recently published or unpublished trials meeting the inclusion criteria.

All searches will be carried out using the Preferred Reporting Items for Systematic Reviews and Meta-Analyses guidance of Population, Intervention, Comparator and Outcome method as listed in the table below (table 2).

The Zotero application will be deployed to retrieve, screen for duplicates and reference all articles.

\section{STUDY RECORDS}

\section{Data management/selection process}

Two authors independent of each other shall screen titles and abstracts retrieved using the search strategy and those from additional sources to select those studies that meet the inclusion criteria outlined above. Thereafter, the full text articles will be retrieved and

Table 2 PICO table

\begin{tabular}{llll}
\hline Population & Intervention & Comparator & Outcomes \\
\hline All patients with diabetes & All forms of mobile health & Standard of care management Improvement in glycaemic \\
mellitus in the africa & (mhealth) implementation in & for diabetes mellitus in the & control measured using mean \\
& the management of diabetes & africa, based on accepted & glycated haemoglobin (hba1c) \\
& mellitus and its complications. & who and International & and percentage changes in \\
& & Diabetes Federation (IDF) & hba1c. \\
& & guidelines.
\end{tabular}


independently assessed for eligibility again by the two authors. A third reviewing author will be brought in to resolve any disagreement between the two authors by discussion. Selected studies will be included for data extraction and analysis.

\section{Data collection process}

A Microsoft word/Google doc-based form will be designed and standardised for the extraction of data, assessment of study quality and synthesis of evidence. A pilot test will be done using 3-5 studies.

Data to be extracted from the articles selected will include the names of authors; year of publication; country of research; sample sizes of both study and control groups; drug treatments used in each study; particular mHealth intervention used in study with the strategy of delivery and functionalities involved; type and durations of studies, study methodology; outcomes and times of measurement including mean baseline glycaemic control using HbA1c or fasting plasma glucose, the mean glycaemic control at the end of study using $\mathrm{HbAlc}$ and fasting plasma glucose, the percentage change in HbAlc, number of hypoglycaemic events and hyperglycaemic crises, quality of life and body mass index (BMI) changes; harms including loss of privacy and inappropriate clinical events; cost of implementation; and indicators of acceptability of intervention information for assessment of risk of bias.

\section{RISK OF BIAS IN SELECTED STUDIES}

Two authors will independently assess the risk of bias in selected studies by following the recommendations of the International Cochrane Collaboration as below given. ${ }^{14}$

1. Concealment of treatment allocation: was the allocated treatment adequately concealed from study participants and clinicians and other healthcare or research staff at the enrolment stage?

2. Blinding: were the personnel assessing outcomes and analysing data sufficiently blinded to the intervention allocation throughout the trial?

3. Completeness of outcome data: were participant exclusions, attrition and incomplete outcome data adequately addressed in the published report?

4. Selective outcome reporting: is there evidence of selective outcome reporting and might this have affected the study results?

5. Other sources of bias: was the trial apparently free of any other problems that could produce a high risk of bias?

A third review author will be brought in where there are disagreements between the two reviewing authors to resolve them by discussion.

\section{DATA SYNTHESIS}

We shall begin with a narrative synthesis which shall describe the following:
1. The intervention used in the studies described (such as individual behaviour change, chronic disease selfmanagement, clinic appointment reminders or clinical diagnostic aid).

2. The mHealth category used and the mobile phone functionalities deployed.

3. The characteristics of the sample population.

4. The expected outcomes especially changes in HbA1c, number of hypoglycaemic events and hyperglycaemic crises, quality of life and BMI changes.

Risk ratios for dichotomous outcomes and standardised mean differences for continuous outcomes shall be calculated to provide summaries of the intervention effects with extracted data.

\section{Statistical analyses}

We will synthesise data by carrying out measurement of effects for the outcomes of the included studies, extracting means and SD rather than calculated effect size. Where multiple outcomes are provided, we will place them in categories that correspond to the type of outcome. Preference will be given to extraction of data that is presented in most acceptable formats requiring the least inputs or inference from authors.

However, this will depend on the characteristics of the eligible studies retrieved and if they employed the same intervention and categories of mHealth. Where this is feasible a statistical analysis to summarise the results of trials using a random effect meta-analysis will be done using Stata V.16.1 (StataCorp) or SPSS V.22 (SPSS), with standardised mean differences for continuous outcomes and risk ratios for binary outcomes where indicated. Each outcome will have a two-sided $\mathrm{p}$ value and a $95 \%$ CI.

\section{Subgroup analysis and investigation of heterogeneity}

Subgroups of data may be created and analysed where heterogeneity is statistically significant depending on the characteristics of the eligible studies retrieved and if they employed the same intervention and categories of mHealth.

Forms of mHealth being considered include voice calls and short messaging services also known as SMS, as well as more complex functionalities and applications such as GPS, GPRS and also third and fourth generation telecommunications 3G, 4G and Bluetooth technology either as part of the mobile phones or as independent wireless devices.

We will assess heterogeneity between studies using both $\chi^{2}$ and $\mathrm{I}^{2}$ and $\mathrm{Q}$ statistics where appropriate. We will compare the pooled study estimates between subgroups defined by study-level characteristics, and use sensitivity analyses to determine the potential sources of heterogeneity.

We will assess for publication bias using a combination of Egger's weighted regression method and Begg's rank correlation as appropriate, depending on whether the outcomes are continuous or dichotomous, respectively. 


\section{DATA STATEMENT}

An application to register this protocol online with the International prospective register of systematic reviews (PROSPERO) has been submitted, and in due course data can be assessed at (https://www.crd.york.ac.uk/ prospero/, registration no. CRD42021218674).

Any amendment to this protocol will require re-registration, republication and updating of the necessary sections.

\section{ETHICS AND DISSEMINATION}

No ethical approval will be required since only published data will be used.

The findings of this review will be published in a peerreviewed journal and probably presented if accepted at a suitable medical conference.

\section{Author affiliations}

${ }^{1}$ Internal Medicine, University of Uyo Teaching Hospital, Uyo, Akwa Ibom, Nigeria

${ }^{2}$ Médecine sociale et préventive-Option: Santé Mondiale, École de Santé Publique, Université de Montréal, Montréal, Quebec, Canada

${ }^{3}$ Centre de Recherche du Centre Hospitalier Universitaire de Montréal (CRCHUM), Montréal, Quebec, Canada

${ }^{4}$ Internal Medicine, University of Uyo, Uyo, Akwa Ibom, Nigeria

${ }^{5}$ Department of Anesthesiology and Critical Care, Stellenbosch University Faculty of Medicine and Health Sciences, Cape Town, Western Cape, South Africa

${ }^{6}$ Human Physiology, Ahmadu Bello University, Zaria, Kaduna, Nigeria

${ }^{7}$ Internal Medicine, Nnamdi Azikiwe University, Awka, Anambra, Nigeria

${ }^{8}$ Internal Medicine, Nnamdi Azikiwe University Teaching Hospital, Nnewi, Anambra,

Nigeria

${ }^{9}$ Internal Medicine, Kaduna State University, Kaduna, Kaduna State, Nigeria

${ }^{10}$ Medicine, University of Cape Town, Rondebosch, Western Cape, South Africa

${ }^{11}$ Internal Medicine, Federal Medical Center Lokoja, Lokoja, Nigeria

${ }^{12}$ Chemical Pathology and Immunology, Kaduna State University, Kaduna, Nigeria

${ }^{13}$ Chemical Pathology, Barau Dikko Teaching Hospital Kaduna, Kaduna, Nigeria

\section{Twitter Franklin Okechukwu Dike @n/a}

Acknowledgements We would like to thank all the reviewers for their timely and constructive inputs to this manuscript.

Contributors All authors contributed substantially to the design of this protocol, drafting of the original version, revision and approval of this major revision. They also agree to share the responsibility for accountability. All authors: conceptualisation, writing of manuscript, reading and editing of manuscript.

Funding The authors have not declared a specific grant for this research from any funding agency in the public, commercial or not-for-profit sectors.

Competing interests None declared.

Patient and public involvement Patients and/or the public were not involved in the design, or conduct, or reporting, or dissemination plans of this research.
Patient consent for publication Not applicable.

Provenance and peer review Not commissioned; externally peer reviewed.

Open access This is an open access article distributed in accordance with the Creative Commons Attribution Non Commercial (CC BY-NC 4.0) license, which permits others to distribute, remix, adapt, build upon this work non-commercially, and license their derivative works on different terms, provided the original work is properly cited, appropriate credit is given, any changes made indicated, and the use is non-commercial. See: http://creativecommons.org/licenses/by-nc/4.0/.

ORCID iD

Franklin Okechukwu Dike http://orcid.org/0000-0003-4773-520X

\section{REFERENCES}

1 WHO Global Observatory for eHealth, World Health Organization. MHealth: new horizons for health through mobile technologies. [Internet]. Geneva: World Health Organization, 2011. Available: http:// www.who.int/goe/publications/goe_mhealth_web.pdf [Accessed 06 Oct 2020].

2 GSM Assoc. African mobile Observatory 2011 driving economic and social development through mobile services, 2011.

3 NW 1615 L. St, Suite 800Washington, Inquiries D 20036USA202419-4300 | M-857-8562 | F-419-4372 | M. Basic mobile phones more common than smartphones in sub-Saharan Africa [Internet]. Pew Research Center's Global Attitudes Project, 2018. Available: https:// www.pewresearch.org/global/2018/10/09/majorities-in-sub-saharanafrica-own-mobile-phones-but-smartphone-adoption-is-modest/ [Accessed 05 Oct 2020].

4 Smartphone and Mobile Internet Penetration In Africa And Globally [Internet]. GeoPoll, 2019. Available: https://www.geopoll.com/blog/ smartphone-mobile-internet-penetration-africa/ [Accessed 05 Oct 2020].

5 Frenk J, Bobadilla JL, Sepuúlveda J, et al. Health transition in middle-income countries: new challenges for health care. Health Policy Plan 1989;4:29-39.

6 Osei K. Global epidemic of type 2 diabetes: implications for developing countries. Ethn Dis 2003;13:S102-6.

7 Young LR, Nestle M. The contribution of expanding portion sizes to the US obesity epidemic. Am J Public Health 2002;92:246-9.

8 Kengne AP, June-Rose McHiza Z, Amoah AGB, et al. Cardiovascular diseases and diabetes as economic and developmental challenges in Africa. Prog Cardiovasc Dis 2013;56:302-13.

9 Shan R, Sarkar S, Martin SS. Digital health technology and mobile devices for the management of diabetes mellitus: state of the art. Diabetologia 2019;62:877-87.

10 Bonoto BC, de Araújo VE, Godói IP, et al. Efficacy of mobile Apps to support the care of patients with diabetes mellitus: a systematic review and meta-analysis of randomized controlled trials. JMIR Mhealth Uhealth 2017;5:e4.

11 World Health Organization. Diagnosis and management of type 2 diabetes (HEARTS-D). (WHO/UCN/NCD/20.1). Geneva, 2020.

12 Iribarren SJ, Cato K, Falzon L, et al. What is the economic evidence for mHealth? A systematic review of economic evaluations of mHealth solutions. PLoS One 2017;12:e0170581.

13 Lewis TL, Wyatt JC. mHealth and mobile medical Apps: a framework to assess risk and promote safer use. J Med Internet Res 2014;16:e210.

14 PROSPERO - Register Guidance Notes [Internet]. Available: https:// www.crd.york.ac.uk/PROSPERO/guidance/risk_of_bias.asp [Accessed 09 Oct 2020]. 